Chirurg 2015 · 86:186

DOI 10.1007/s00104-015-2988-y

Online publiziert: 30. Januar 2015

c) Springer-Verlag Berlin Heidelberg 2015
F. Benedix · C.J. Bruns

Klinik für Allgemein-, Viszeral- und Gefäßchirurgie, Klinik für Chirurgie,

Universitätsklinikum Magdeburg A.ö.R., Magdeburg

\title{
Einfluss bariatrischer Eingriffe auf die nichtalkoholische Fettlebererkrankung
}

\section{Vergleich des Roux-en-Y-Magenbypass mit dem adjustierbaren Magenband}

\section{Originalpublikation}

Caiazzo R, Lassailly G, Leteurtre E et al (2014) Roux-en-Y gastric bypass versus adjustable gastric banding to reduce nonalcoholic fatty liver disease: a 5-year controlled longitudinal study. Ann Surg 260:893-898

\section{Hintergrund und Fragestellung}

Die nichtalkoholische Fettlebererkrankung (NAFLD) gilt als hepatische Manifestation des metabolischen Syndroms und ist eng mit der Adipositas assoziiert. Die effektivste Behandlung besteht in einer Gewichtsreduktion. Die bariatrische Chirurgie repräsentiert die aktuell einzige Behandlungsoption, mit deren Hilfe eine signifikante Gewichtsreduktion sowie eine Beeinflussung assoziierter Komorbiditäten erreicht und erhalten werden kann. Ziel der vorliegenden Studie war, erstmalig verschiedene bariatrische Eingriffe hinsichtlich ihrer Effektivität auf die NAFLD zu untersuchen.

\section{Methoden}

In der unizentrischen prospektiven Studie erfolgte der direkte Vergleich des RouxY-Magenbypass (RYGB) mit dem adjustierbaren Magenband (AGB). Neben der Untersuchung klinischer und laborchemischer Parameter erfolgte die histologische Aufarbeitung von Punktionshistologien der Leber, die sowohl intraoperativ als auch nach einem bzw. 5 Jahren postoperativ entnommen wurden.

\section{Ergebnisse}

Bei insgesamt 681 Patienten erfolgte die Durchführung eines RYGB, bei 555 wurde ein AGB implantiert. Eine verwertbare Leberbiopsie war bei 97,2\% verfügbar. Insgesamt $86 \%$ der Patienten wiesen zum Zeitpunkt der Operation eine NAFLD auf. Die Follow-up-Rate nach 12 Monaten betrug 95,2\%, 5 Jahre nach dem Eingriff immerhin $80,1 \%$. Leberbiopsien wurden bei 47,2\% bzw. 68,9\% der untersuchten $\mathrm{Pa}$ tienten nach einem bzw. 5 Jahren entnommen und analysiert.

Nach beiden Eingriffen zeigten sich ein signifikanter Gewichtsverlust sowie eine Verbesserung der metabolischen und hepatischen Zielparameter. In einigen Fällen konnte eine komplette Remission der NAFLD bzw. Remission einer initialen Fibrose nachgewiesen werden.

Im direkten Vergleich beider Operationsverfahren fand sich ein signifikanter Vorteil sowohl bezüglich des Gewichtsverlustes als auch hinsichtlich einer Verbesserung der metabolischen sowie hepatischen Zielparameter für den RYGB. In einer multivariaten Analyse wurde der stärkere Effekt des RYGB vornehmlich auf den höheren Gewichtsverlust zurückgeführt.

\section{Fazit}

Die vorliegende Studie vergleicht erstmalig die Effektivität verschiedener bariatrischer Operationen auf die NAFLD.
Hervorzuheben sind die hohe Patientenzahl, der lange Nachbeobachtungszeitraum sowie die akzeptable Follow-upRate. Letztere ist insbesondere zu würdigen, da eine invasive Untersuchung Teil der Nachsorge war. Die Autoren bestätigen den positiven Effekt der bariatrischen Chirurgie auf die NAFLD. Darüber hinaus konnten sie nachweisen, dass der RYGB effektiver und schneller eine Verbesserung der hepatisch-metabolischen Komorbidität bewirkt. Auf der Basis ihrer Ergebnisse empfehlen die Autoren die Durchführung eines RYGB bei morbider Adipositas und fortgeschrittener NAFLD. Ein interessanter Aspekt der Studie ist die durchweg positive Beeinflussung der fortgeschrittenen Lebererkrankung, insbesondere der Leberfibrose. Neben der Untersuchung des Langzeiteffektes der bariatrischen Chirurgie auf die NAFLD sollte dieser Aspekt sowie ein möglicher präventiver Effekt auf die Entwicklung eines hepatozellulären Karzinoms Fokus zukünftiger Studien sein.

\section{Korrespondenzadresse}

PD Dr. F. Benedix

Klinik für Allgemein-, Viszeral- und Gefäßchirurgie, Klinik für Chirurgie, Universitätsklinikum Magdeburg A.ö.R., Leipziger Str. 44, 39120 Magdeburg frankbenedix@gmx.de

Interessenkonflikt. F. Benedix und C.J. Bruns geben an, dass kein Interessenkonflikt besteht. 